\title{
Doxycycline-regulated growth hormone gene expression system for swine
}

B.C. Jiang ${ }^{1}$ D. B. Yu ${ }^{1}$, L J. Wang ${ }^{1}$ F.L. Dong ${ }^{1}$ H.A. Kaleri ${ }^{2}$ X. G. Wang N. Ally ${ }^{1}$, J. Li $^{1}$ and H.L. Liu ${ }^{1}$

'Department of Animal Breeding \& Genetics, College of Animal Science \& Technology, Nanjing Agricultural University, Nanjing, China

${ }^{2}$ Department of Animal Breeding and Genetics, Faculty of Animal Husbandry and Veterinary Sciences, Sindh Agriculture University, Tando Jam, Pakistan ${ }^{3}$ Department of Animal Breeding \& Genetics, College of Animal Science \& Technology, Xinjiang Agricultural University, Xinjiang, China

Corresponding author: H.L. Liu

E-mail: liuhonglin@263.net

Genet. Mol. Res. 11 (3): 2946-2957 (2012)

Received September 27, 2011

Accepted February 6, 2012

Published July 10, 2012

DOI http://dx.doi.org/10.4238/2012.July.10.5

\begin{abstract}
Although growth hormone gene transgenic animals are much larger than normal animals, they manifest differences that have adverse effects on survival due to overexpression of growth hormone. We developed a stable pig embryonic fibroblast cell line expressing pig growth hormone (pGH) using the Tet-On system, with which we can conditionally manipulate expression of pGH in vivo. Inducible expression of $\mathrm{pGH}$ was achieved by combining reserve Tet-controlled transcriptional activator and tetracycline-responsive element in a single plasmid. The mRNA expression of pGH was significantly increased compared to the non-induced group by about 10 -fold. The controlled
\end{abstract}


secretion of pGH induced by doxycycline was further tested in stably transfected cells. We conclude that inducible GH expression can be achieved in pig embryonic fibroblasts.

Key words: Growth hormone; Tet-On system; Lentiviral vector; Doxycycline

\section{INTRODUCTION}

Growth hormone $(\mathrm{GH})$ plays an important role in the regulation of growth and metabolism (Kolb, 1977). It promotes growth of the skeletal system and facilitates the incorporation of amino acids during protein synthesis (Madsen et al., 1983; Mayo et al., 1983). Palmiter et al. (1982) developed the first GH transgenic mice whose growth was dramatically accelerated. This approach motivated many countries to invest much capital in the study of GH transgenic farm animals, aimed at enhancing production performance (Krasnov et al., 1999; Hens et al., 2000; Lipinski et al., 2003). These GH transgenic animals showed higher growth rates and daily weight gain compared to controls, but the overexpression of the exogenous GH gene brought forth a host of pathophysiological abnormalities and higher early mortality (Orian et al., 1989; Barton et al., 1992). Consequently, a method of keeping the exogenous gene at a slightly higher level is needed to produce healthy and high-yielding GH transgenic animals. For this purpose, a technique that can conditionally manipulate the expression of the GH gene in a growth stage-specific fashion would be desirable.

The Tet-On system, which was developed by Gossen et al. in 1995, has been widely used to tightly control foreign gene expression in a variety of mammalian cells in culture as well as in a large amount throughout the whole animal (Bockamp et al., 2008; Takiguchi et al., 2010). Many improvements have been made to the original version of this system. One is the use of an improved version of the reverse tetracycline (Tet)-controlled transactivator protein (rtTA), called rtTA-Advanced, which is more sensitive to doxycycline (Dox) and yields lower background expression than the original rtTA version. Another improvement is the construction of a bidirectional tetracycline-responsive element (TRE)-Tight plasmid that can be used to inducibly express two genes (a combination of a reporter with one or two genes of interest). To rapidly generate multiple stable cell lines with regulatable exogenous gene expression and ease injury to the primary host cells, the Tet-On elements were incorporated into a single plasmid. This has been demonstrated to closely regulate cDNA and shRNA expression in several cell lines (Johansen et al., 2002; Wiederschain et al., 2009).

The lentiviral vector can effectively deliver heterologous sequences to both dividing and nondividing mammalian cells and mediate stable in vivo gene transfer into terminally differentiated cells (Naldini et al., 1996). It provides stable, long-term expression of a target gene beyond that offered by traditional adenoviral-based systems (Dull et al., 1998). The third-generation lentiviral expression system can produce a pseudotyped virus with a broadened host range and include numerous safety features designed to enhance its biosafety. Once integrated into the target cells, the lentiviral genome is no longer capable of producing a packageable viral genome (Dull et al., 1998). 
In this study, a single-plasmid lentiviral Tet-On inducible pGH gene (pLenti-TRETight-BI-GH-rtTA-Advanced) expression system was generated to obtain stable transfectants in pig embryo fibroblast (PEF) cells. The inducible pGH expression PEF cell line (I-pGH PEF cell line) was used for transgenic nuclear transfer with the aim of obtaining a healthy pGH transgenic pig breed with a fast growth rate and high feed-conversion efficiency.

\section{MATERIAL AND METHODS}

\section{Plasmids}

Total RNA was isolated from large Yorkshire pig pituitary glands using the TRIzol reagent (Invitrogen) according to the manufacturer protocol. Reverse transcription was performed with $2 \mu \mathrm{g}$ total RNA following the TaKaRa protocol. The pig GH sequence was amplified from cDNA by PCR using primer set 1 (Table 1), and the PCR product was then doubledigested and inserted into pTRE-Tight-BI (Clontech) in MSCI to obtain pTRE-Tight-BI-GH. To construct pTRE-Tight-BI-GH-rtTA-Advanced, the rtTA-Advanced fragment was amplified by PCR from the pTet-On advanced (Clontech) with primer set 2 (Table 1). This fragment was subcloned into the plasmid pTRE-Tight-BI-GH in MSCII using the EcoRI and BglII restriction sites. Finally, a GH single vector Tet-On Advanced Inducible Gene Expression system was constructed (Figure 1A).

\begin{tabular}{|c|c|c|c|c|}
\hline No. & Gene & Restriction enzyme & Primer sequence & Amplicon size (bp) \\
\hline \multirow[t]{2}{*}{1} & GH & BamHI & F: 5'-GTGGATCCCTCAGCTCACCGGC-3' & 696 \\
\hline & & SalI & R: 5'-CAACAGA $\boldsymbol{G T C G A C C A G C A A C T A G A A - 3 '}$ & \\
\hline \multirow[t]{2}{*}{2} & rtTA-Advanced & & F: 5'-TGACCTCCATAGAAGACACC-3' & 776 \\
\hline & & Bgla & R: 5'-ATGTCT $\boldsymbol{A G A T C T T T A C T T A G T T A C C - 3 '}$ & \\
\hline \multirow[t]{2}{*}{3} & TRE-Tight-BI-GH-rtTA & SpeI & F: 5'-CTAG $\boldsymbol{A C T} \boldsymbol{A} \boldsymbol{G T C C T G A C G T C G G C A G T G A A A A - 3 '}$ & 6548 \\
\hline & -Advanced fragment & Saca & 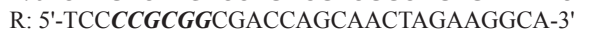 & \\
\hline \multirow[t]{2}{*}{4} & Lentivector backbone & & F: 5'-GCGACTTCCAGTTCAA-3' & 1753 \\
\hline & & SpeI & R: 5'-CTAG $\boldsymbol{A C T} \boldsymbol{A} \boldsymbol{G T C T C C A C C T T C T T C T T C T A T - 3 '}$ & \\
\hline \multirow[t]{2}{*}{5} & GH & & F: 5'-GTTCCTCAGCAGGGTCTT-3' & 260 \\
\hline & & & R: 5'-CCCGCAGGTATGTCTCA-3' & \\
\hline \multirow[t]{2}{*}{6} & HPRT2 & & F: 5'-CTGGCAAAACAATGCAAACCT-3' & 73 \\
\hline & & & R: 5'-AAGCTTGCAACCTTGACCATCT-3' & \\
\hline \multirow[t]{4}{*}{7} & TRE-mod & & F: 5'-ACAAGGAAACTCGCTCAAA-3' & 421 \\
\hline & & & R: 5'-GGCATAGAATCGGTGGTAG-3' & \\
\hline & GAPDH & & F: 5'-CTGCCCCTTCTGCTGATGC-3' & 151 \\
\hline & & & R: 5'-TCCACGATGCCGAAGTTGTC-3' & \\
\hline
\end{tabular}

The expression cassette TRE-Tight-BI-GH-rtTA-Advanced fragment was amplified by PCR from pTRE-Tight-BI-GH-rtTA-Advanced with primer set 3 (Table 1). The product was subcloned into pMD 19-T vector in the T-cloning site. The lentivector backbone was derived from plasmid pLenti6-V5-GW-LacZ (Invitrogen) by PCR using primer set 4 (Table 1). Finally, the TRE-Tight-BI-GH-rtTA-Advanced fragment from pMD19-TRE-Tight-BI-GHrtTA-Advanced was subcloned into the lentivector in place of the RRE fragment to generate the plasmid pLenti-TRE-Tight-BI-GH-rtTA-Advanced (Figure 1B). 
A

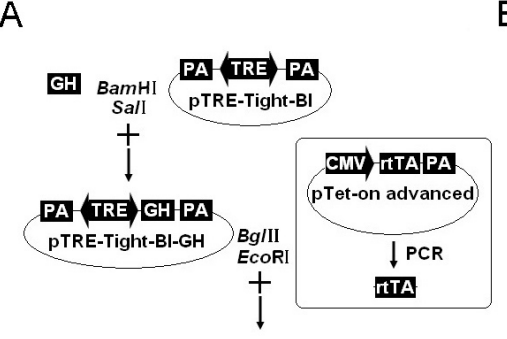

PATITA(TRE) GH PA

pTRE-Tight-BI-GH-rTTA
B
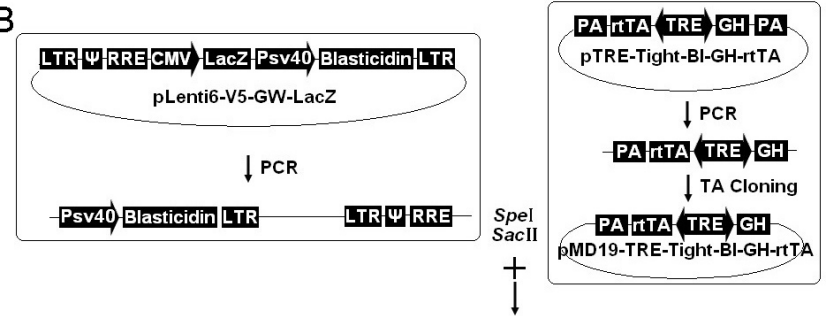

LTR PRREPAITAA(TRE) GHPSV40 Blasticidin LTR

pLenti-TRE-Tight-BI-GH-rTTA

Figure 1. Single-plasmid lentiviral Tet-On inducible pGH gene expression system for stable expression in mammalian cells. A. Schematic description for the construction of the pTRE-Tight-BI-GH-rtTA-Advanced vector used to detect whether the single-plasmid system can closely regulate pGH gene expression in PEF cells. The plasmid includes pGH and rtTA-Advanced cDNA derived by TRE mod-dependent minCMV-1 and minCMV-2, respectively. B. Schematic representation of the lentiviral plasmid used to mediate the TRE-Tight-BI-GH-rtTAAdvanced fragment stably transfected into PEF cells. The lentivector backbone includes 5'LTR, 3'LTR, HIV-1 psi $(\psi)$ packaging signal and HIV-1 Rev response element (RRE), which permits viral packaging and reverse transcription of the viral mRNA. In addition, the lentivector backbone contains the SV40 early promoter, and the blasticidin resistance gene allows selection of stably transduced mammalian cell lines. The right part of the figure illustrates that only the 5'-region of the TRE-Tight-BI-GH-rtTA-Advanced fragment contains an SV 40 polyadenylation signal sequence.

\section{Production of lentiviral stocks}

Lentiviral vectors were generated by co-transfecting $293 \mathrm{FT}$ cells at $80-90 \%$ confluency with the lentivirus expression plasmid pLenti-TRE-Tight-BI-GH-rtTA-Advanced and the packaging plasmids pLP 1, pLP 2 and pLP/VSVG, using Lipofectamine 2000 (Invitrogen) following a standard lipid-mediated transfection procedure. Viral particles were harvested 48$72 \mathrm{~h}$ after transfection and centrifuged at $3000 \mathrm{rpm}$ for $15 \mathrm{~min}$ to pellet the cell debris. They were then filtered using Millex-HV 0.45- $\mu \mathrm{m}$ PVDF filters (Millipore). To obtain a higher titer, centrifugation at $50,000 \mathrm{~g}$ for $2 \mathrm{~h}$ at $4^{\circ} \mathrm{C}$ was performed. The pellet was resuspended in Dulbecco's modified Eagle's medium (DMEM; Gibco) for $4 \mathrm{~h}$ on ice and then stored at $-80^{\circ} \mathrm{C}$ until used. Viral stocks were titered by the Lenti-XTM qRT-PCR Titration kit (Clontech).

\section{Primary cultures}

All experiments in this study were performed using primary fetal fibroblast cells isolated from a 33-day-old large Yorkshire fetus. The head, tail, extremities and viscera of this fetus were excised under sterile conditions, and other portions of the fetus were then aseptically cut into small pieces using micro-scissors in sterile phosphate-buffered saline (PBS; Gibco). After sterilization and trypsinization, the pig fetus embryonic fibroblast cells were separated freely and grown in T75 flasks containing DMEM (Gibco) supplemented with $10 \%$ fetal calf serum. After 5-6 days, the cells attached to the bottom of the flasks and formed a monolayer. Within 13-15 days, the cells were $90 \%$ confluent and were transferred to new flasks. After mild trypsinization, the cells were subcultured once a week. They were normally subcultured two times before the experiment was performed. 


\section{Vector transfection and semiquantitative RT-PCR}

One day before transfection, PEF cells were seeded at a density of $5 \times 10^{5}-10 \times$ $10^{5}$ per well in 6-well culture plates and were $70-90 \%$ confluent at the time of transfection. The confluent cells were randomly divided into three groups. In Group 1, cells were treated with Lipofectamine 2000 for only $6 \mathrm{~h}$ and then grown in medium supplemented with $1 \mu \mathrm{g} /$ mL Dox. In Group 2, cells were transduced with $4 \mu \mathrm{g}$ pTRE-Tight-BI-GH-rtTA-Advanced vector, and $6 \mathrm{~h}$ later, the medium with transfection complexes was replaced with fresh medium without Dox. In Group 3, cells were transduced with $4 \mu \mathrm{g}$ pTRE-Tight-BI-GH-rtTAAdvanced vector, and $6 \mathrm{~h}$ later, the medium with transfection complexes was replaced with medium containing $1 \mu \mathrm{g} / \mathrm{mL}$ Dox. The total RNA of the three samples in each group was collected and treated with DNase I to remove possible contaminating plasmid DNA. Semiquantitative RT-PCR was performed using the HPRT2 gene as an inner control. Primer sets 5 and 6 (Table 1) were used for GH and HPRT2 amplification. A total of $10 \mu \mathrm{L}$ of each PCR product were resolved on a $2.5 \%$ agarose gel, stained with ethidium bromide and photographed with Quantity One (Bio-Rad). The PCR products were then quantified by scanning densitometry using the Image $\mathrm{J}$ software (NIH).

\section{Viral infection and stable cell line}

Cells at approximately $25 \%$ confluency were treated with medium containing varying concentrations of blasticidin $(0,2,4,6,8$, and $10 \mu \mathrm{g} / \mathrm{mL})$. The selective media were replenished every 4 days, and the appropriate concentration of blasticidin that kills the cells within 10-14 days after adding the antibiotic was determined. Cells were seeded in 6-well culture plates and were $25 \%$ confluent before transduction. Transduction was performed by adding $50 \mu \mathrm{L}$ lentiviral stock and $12 \mathrm{mg}$ polybrene per well. The medium was replaced with medium containing $4 \mu \mathrm{g} / \mathrm{mL}$ blasticidin $72 \mathrm{~h}$ later. The medium was replaced every 4 days until non-transgenic cells were killed. PCR was used to analyze colonies of integration with lentiviral constructs.

\section{Detection of inducible GH expression by ELISA}

The positive colonies were subcultured in 6-well plates and treated with media supplemented with Dox $(0,24$, and $48 \mathrm{~h})$. The concentration of $\mathrm{GH}$ in the conditioned medium was determined by a commercially available porcine growth hormone ELISA kit (USCN LIFE). ELISA was performed according to the operating instructions of the manufacturer. Briefly, the samples or standards were added to microtiter plate wells coated with a biotin-conjugated GH polyclonal antibody preparation specific for $\mathrm{GH}$, and avidin conjugated to horseradish peroxidase was added to each microplate well and incubated. A TMB substrate solution was then added to each well. Only those wells that contained GH, biotin-conjugated antibody and enzyme-conjugated avidin exhibited a change in color. The enzyme-substrate reaction was terminated by the addition of a sulfuric acid solution. The color change was measured spectrophotometrically at a wavelength of $450 \pm 2 \mathrm{~nm}$. 


\section{Karyotype analysis}

Karyotype analysis of the I-pGH PEF cell line was performed with a DAPI Karyotyping kit (Genmed) according to the manufacturer protocol.

\section{Statistical analysis}

Data are presented as means \pm SE. Statistical significance between two groups was determined by the Student $t$-test. A P value of $<0.05$ was considered statistically significant.

\section{RESULTS AND DISCUSSION}

\section{The transgenic plasmid system}

Circumventing the difficulties of creating a double-stable Tet-On Advanced cell line that contains integrated copies of the regulatory vector (pTet-On Advanced) and response vector (pTRE-Tight) is time-consuming; hence, we aimed to establish a system for transgenesis by one-step stable transfection. First, we inserted GH and the rtTA-Advanced gene cassette into multiple cloning sites I and II of a bidirectional TRE-Tight plasmid (pTREtight-BI), respectively. In addition, the key fragment of the newly constructed GH inducible expression vector was subcloned into a lentivector that contained a blasticidin resistance gene for selecting stable transformants (for a schematic description of the construct, see Figure 1A and B). The GH cDNA sequence included its Kozak consensus sequence that improved the expression level and signal peptide encoding region directing the transport of pGH protein. The reason for the absence of the 3'-region SV 40 polyadenylation signal sequence in the TRE-Tight-BI-GH-rtTA-Advanced fragment obtained by PCR amplification was that the transcription of GH can be terminated by an HIV-1 truncated 3'-LTR containing a polyadenylation signal. The map of the constructed plasmid pTRE-Tight-BI-GH-rtTAAdvanced and pLenti-TRE-Tight-BI-GH-rtTA-Advanced is given in Figure 2a and c. Figure $2 \mathrm{a}$ reveals that the two Tet-responsive mini CMV promoters, which control the expression of both GH and the rtTA-Advanced gene, flank the TRE-mod. Figure $2 \mathrm{c}$ shows that the size of the recombinant lentivirus vector was $8334 \mathrm{bp}$, which would not have an effect on virus titers. Recombinant vectors were confirmed by restriction enzyme digestion (Figure $2 b$ and $d$ ) and sequencing (data not shown).

In the course of vector construction, we observed a series of phenomena. First, we had expected to connect the lentivector backbone and TRE-Tight-BI-GH-rtTA-Advanced fragment, both obtained by PCR, together into a plasmid directly. We made several attempts, but failed each time. After TA cloning, the insert was cut with SpeI and SacII and cloned into the lentivector successfully. This could account for the fact that the sticky ends originating from the chemically synthesized primer sequence differed from those of endogenous DNA. Second, TA cloning of large DNA fragments was more difficult than cloning small DNA fragments. On accomplishing this, only three transformants were created. 
A

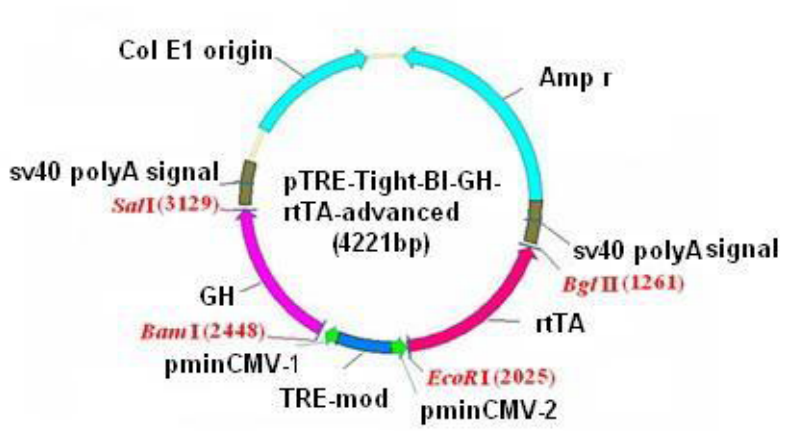

C

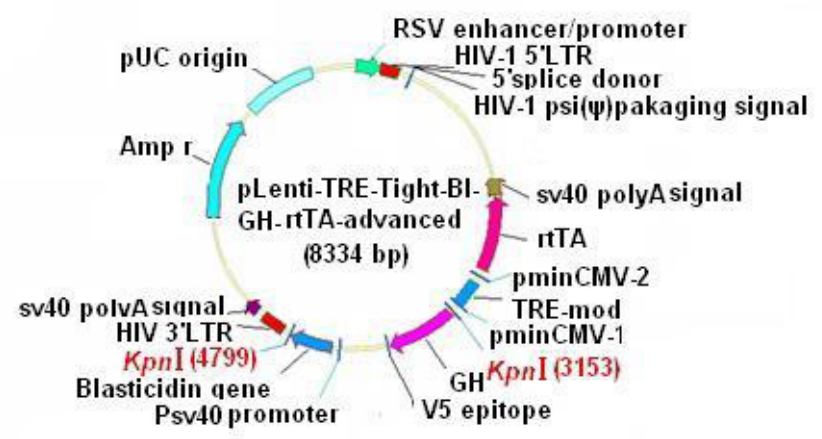

B

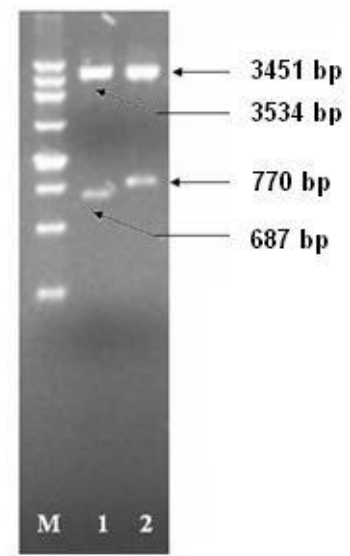

D

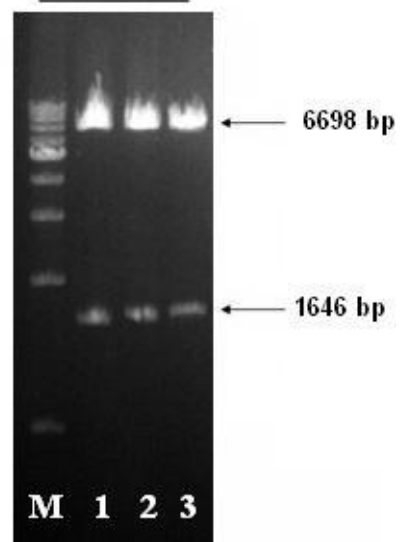

Figure 2. Construction map of pTRE-Tight-BI-GH-rtTA-Advanced and pLenti-TRE-Tight-BI-GH-rtTAAdvanced vectors. A. Information on the pTRE-Tight-BI-GH-rtTA-Advanced vector (4221 bp). B. Agarose gel electrophoresis of newly constructed plasmid digested with enzymes (lane 1 was digested with BamHI and SalI, lane 2 was digested with $E c o$ RI and BglII, and lane $M$ shows a 500-bp ladder marker; TaKaRa). C. This panel shows the construction map of the pLenti-TRE-Tight-BI-GH-rtTA-Advanced vector ( $8334 \mathrm{bp})$. D. Digestion result of the pLenti-TRE-Tight-BI-GH-rtTA-Advanced vector with KpnI restriction enzymatic sites (lanes 1, 2 and $3=$ digestion samples (6698 and $1646 \mathrm{bp}$ ) and lane $M=\mathrm{a} 1-\mathrm{kb}$ ladder marker; TaKaRa).

\section{Verification test of the GH gene inducible expression vector}

We examined the ability of Dox to induce pGH expression in cultured PEF cells by semiquantitative RT-PCR. PEF cells were capable of inducing pGH expression by transient transfection with the pTRE-Tight-BI-GH-rtTA-Advanced vector. The optimum concentration for induction in PEF cells had been intended to be $10 \mu \mathrm{g} / \mathrm{mL}$. After $48 \mathrm{~h}$ of induction, transcript levels of Group 3 significantly improved compared to those of the non-transfected (Group 1) and non-induced (Group 2) controls. No obvious difference was found between Groups 1 and 2. These results suggested that our system was more sensitive to Dox and yielded a lower background. In fact, the key fragment had not been inserted into the lentivector until the verification test was finished (Figure 3). 
A

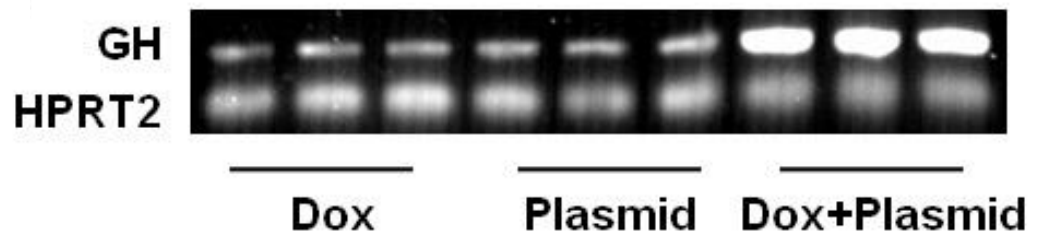

B

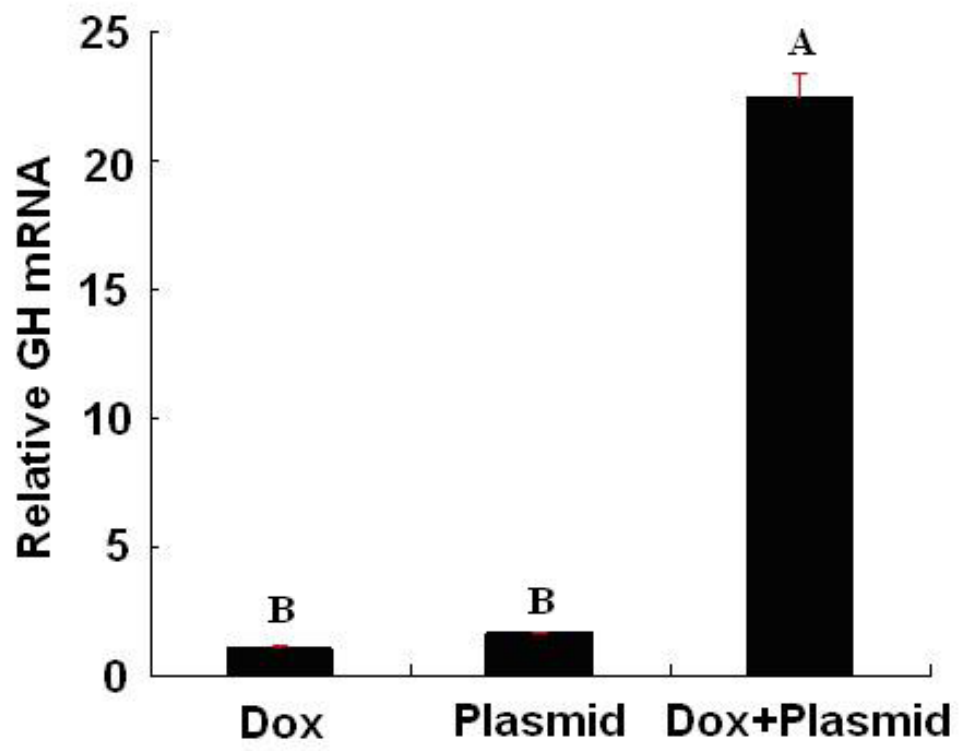

Figure 3. Semiquantitative RT-PCR analyses of pGH gene expression among the Dox, Plasmid and Dox + Plasmid groups. A. Representative agarose gel of photographed RT-PCR products stained with ethidium bromide. B. Histograms obtained from intensity ratios of the GH mRNA RT-PCR product to that of HPRT2 (error bars indicate the standard deviation; different capital letters above columns denote statistically significant differences $\mathrm{P}<0.01 ; \mathrm{N}$ =3). $\mathrm{GH}=$ Growth hormone. Dox $=$ doxycycline.

\section{Dox-inducible pGH gene expression in I-pGH PEF cell line}

At 14 days after selection, four blasticidin-resistant colonies of PEF cells were obtained and analyzed by PCR using primer sets 7 and 8 (Table 1). All four clones were identified to be stably transduced with the lentiviral construct (Figure 4). The colonies were picked and expanded to assay for inducible expression of the pGH protein.

Medium GH concentration was quantified using an ELISA kit. The absorbance of serial dilution stands were measured at $450 \pm 2 \mathrm{~nm}$ and used to make the standard curve, where absorbance was plotted against GH concentration (Figure 5A). The concentration of GH of all samples was calculated according to this standard curve. The GH level at $48 \mathrm{~h}$ after Dox treatment showed was significantly increased compared to that at 0 and $24 \mathrm{~h}$. The mean GH concentration in medium was $9.493 \pm 0.677 \mathrm{ng} / \mathrm{mL}$, which was 2.32- and 2.17-fold higher than that of the 0 and $24 \mathrm{~h}$ groups, respectively. That no significant difference was observed between the 0 and $24 \mathrm{~h}$ groups was unexpected. We speculate that the low expression rate of 
pGH in the 24-h group was primarily due to the insufficient transactivators (rtTA-Advanced protein) generated by basal expression in I-pGH PEF cells. In the traditional double-stable TetOn Advanced cell line, rtTA-Advanced protein expression was driven by the wild-type CMV immediate early promoter from the integrated copies of the regulatory vector and could quickly accumulate enough to activate transcription binding to TRE-mod in the presence of Dox. In our newly established I-pGH PEF cell line, the accumulation of rtTA-Advanced was only driven by the Tet-responsive miniCMV promoters. These promoters lacked the enhancer and flanked the TRE-mod, so the concentration of regulatory protein could not reach the threshold needed to activate Tet-responsive miniCMV promoters in a short time.
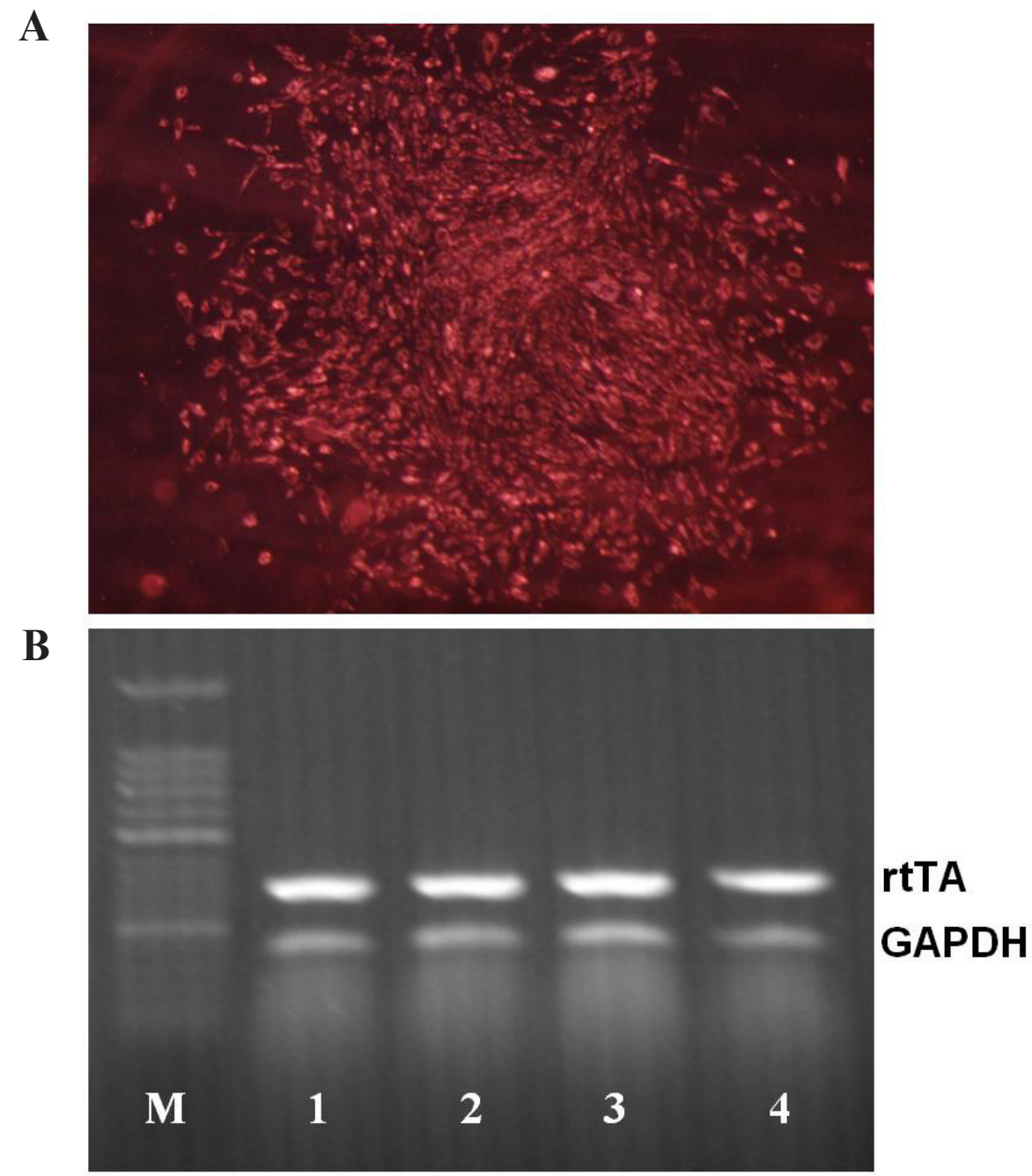

Figure 4. Identification of blasticidin-resistant colonies. A. Representative cell colony under light microscopy. B. PCR analysis of representative blasticidin-resistant clonies containing inserted lentiviral constructs. Lane $M=$ 50-bp DNA ladder marker; lanes 1, 2, 3, and $4=$ PCR product from colonies 1, 2, 3, and 4, respectively. GAPDH were used as control. 
A

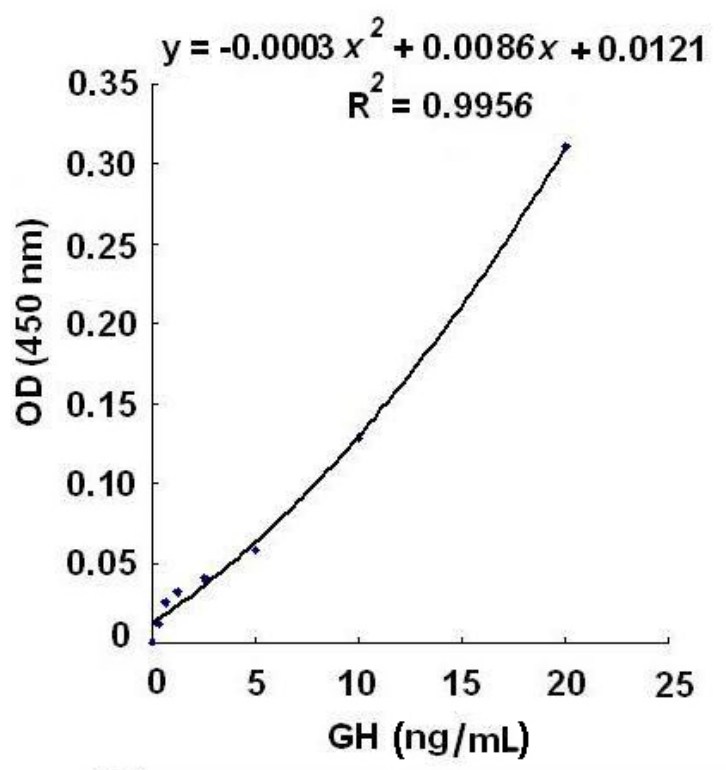

B

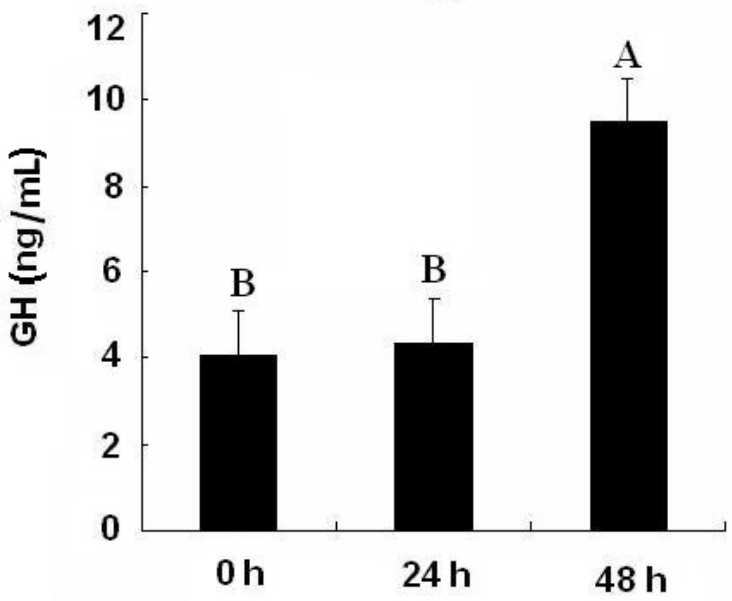

Figure 5. Growth hormone $(\mathrm{GH})$ protein levels were determined using ELISA in the stable transfected cells. A. Standard ELISA curve for the determination of the $\mathrm{pGH}$ protein in medium. The fitting equation " $\mathrm{y}=-0.0003 \mathrm{x} 2$ $+0.0086 \mathrm{x}+0.0121 ; \mathrm{R} 2=0.9956$ " was established by application of a nonlinear regression method. B. The concentration of the pGH protein in medium was plotted against Dox treatment time (error bars indicate the standard deviation; different capital letters denote statistically significant differences $\mathrm{P}<0.01 ; \mathrm{N}=6$ ).

The main significance of generating a fast I-pGH PEF cell line lies in the fact that it can cut down the selection period greatly and minimize harm to host cells. Because the isolation of pig embryonic stem cells is difficult, researchers can only use PEF cells, which have a limited proliferative ability, as the nuclear transfer donor cell. Cells would be badly injured by creating a double-stable Tet-On PEF cell line via double-stable transfection. The integrated copies of the regulatory vector and response vector contained in $\mathrm{F}_{1}$ transgenic animals can be separated in meiotic division. 


\section{Chromosome analysis}

The results for the karyotype of I-pGH-positive cells by chromosomal DAPI-band analysis showed that these cells had a normal karyotype after six passages of subculture. Figure 6 presents the chromosomal analysis. The I-pGH PEF cells showed no change in chromosomal number or morphology. Dick et al. (2011) also found that approximately $95 \%$ of cell lines created by lentiviral vectors showed a normal karyotype. Generally, lentivirus-mediated transgenic cells cannot lead to chromosomal abnormalities.

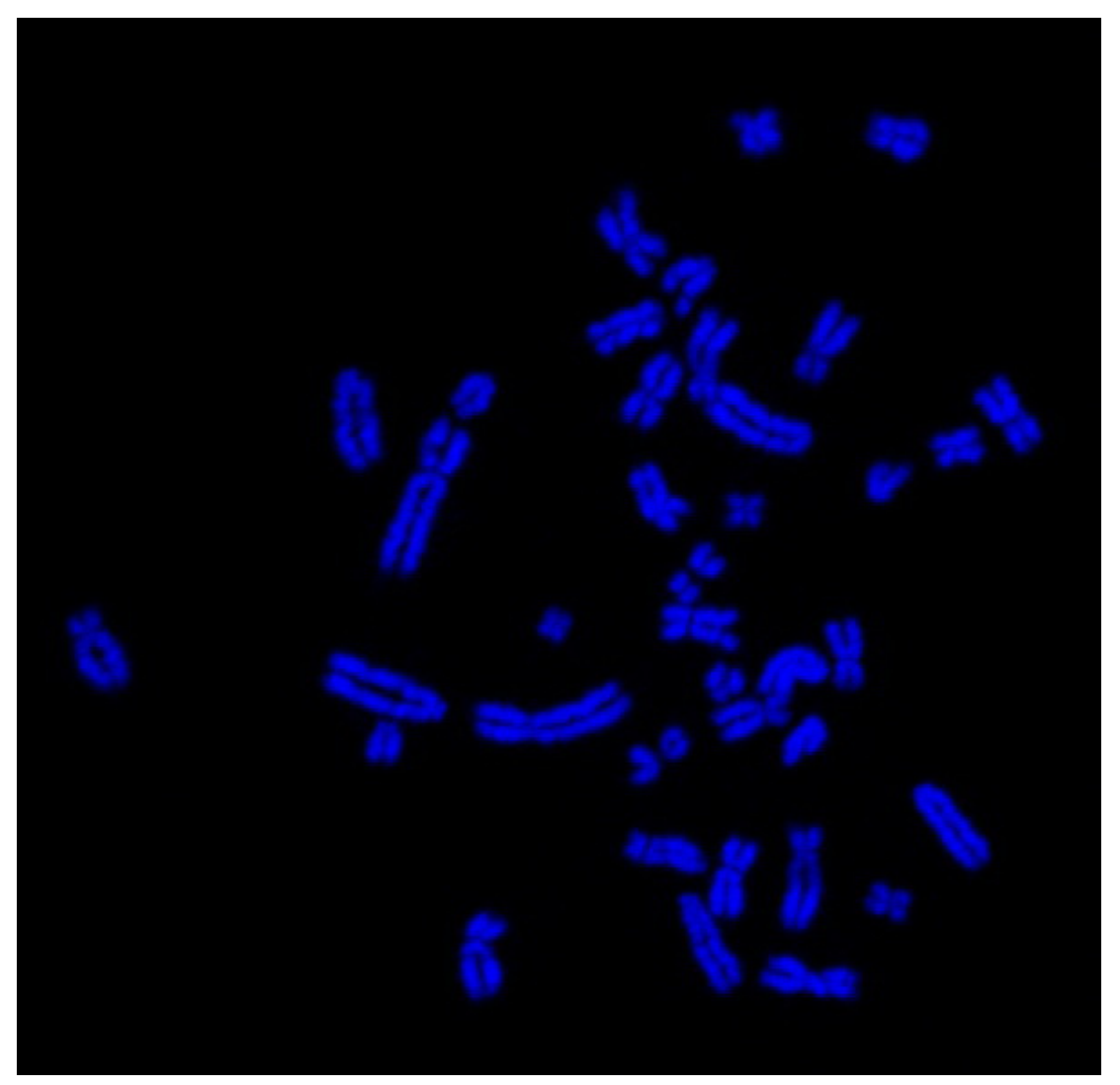

Figure 6. Karyotype analysis of chromosomes from a colony of cells stably integrated by a Dox-regulated GH gene expression system.

\section{CONCLUSION}

In the present study, we showed that the pGH protein can be secreted from I-pGHpositive cells and that its concentration can be conditionally manipulated by Dox. This system should be of great help in producing healthy and high-yielding GH transgenic pigs by somatic cell nuclear transfer. 


\title{
ACKNOWLEDGMENTS
}

\author{
Research supported by grants from Projects of Cultivating New Varieties by Trans- \\ genic Technology (\#2008ZX08006-003).
}

\section{REFERENCES}

Barton JS, Cullen S, Hindmarsh PC, Brook CG, et al. (1992). Growth hormone treatment in idiopathic short stature: a preliminary analysis of cardiovascular effects. Acta Pediatr. Suppl. 383: 35-38.

Bockamp E, Sprengel R, Eshkind L, Lehmann T, et al. (2008). Conditional transgenic mouse models: from the basics to genome-wide sets of knockouts and current studies of tissue regeneration. Regen. Med. 3: 217-235.

Dick E, Matsa E, Young L, Darling D, et al. (2011). Accelerating the generation of human induced pluripotent stem cells by coupling high titre lentivirus and column-based positive selection of hiPSCs. Nat. Protoc. 6: 701-714.

Dull T, Zufferey R, Kelly M, Mandel RJ, et al. (1998). A third-generation lentivirus vector with a conditional packaging system. J. Virol. 72: 8463-8471.

Hens JR, Amstutz MD, Schanbacher FL and Mather IH (2000). Introduction of the human growth hormone gene into the guinea pig mammary gland by in vivo transfection promotes sustained expression of human growth hormone in the milk throughout lactation. Biochim. Biophys. Acta 1523: 161-171.

Johansen J, Rosenblad C, Andsberg K, Moller A, et al. (2002). Evaluation of Tet-on system to avoid transgene downregulation in ex vivo gene transfer to the CNS. Gene Ther. 9: 1291-1301.

Kolb E (1977). Recent findings relating to the importance of growth hormone to both regulation of metabolism and production performance of ruminants (author's transl). Monatsh. Veterinarmed. 32: 230-235.

Krasnov A, Agren JJ, Pitaknen TI and Molsa H (1999). Transfer of growth hormone (GH) transgenes into Arctic charr. (Salvelinus alpinus L.) II. Nutrient partitioning in rapidly growing fish. Genet. Anal. 15: 99-105.

Lipinski D, Jura J, Kalak R, Plawski A, et al. (2003). Transgenic rabbit producing human growth hormone in milk. J. Appl. Genet. 44: 165-174.

Madsen K, Friberg U, Roos P, Eden S, et al. (1983). Growth hormone stimulates the proliferation of cultured chondrocytes from rabbit ear and rat rib growth cartilage. Nature 304: 545-547.

Mayo KE, Vale W, Rivier J, Rosenfeld MG, et al. (1983). Expression-cloning and sequence of a cDNA encoding human growth hormone-releasing factor. Nature 306: 86-88.

Naldini L, Blomer U, Gallay P, Ory D, et al. (1996). In vivo gene delivery and stable transduction of nondividing cells by a lentiviral vector. Science 272: 263-267.

Orian JM, Lee CS, Weiss LM and Brandon MR (1989). The expression of a metallothionein-ovine growth hormone fusion gene in transgenic mice does not impair fertility but results in pathological lesions in the liver. Endocrinology 124: 455-463.

Palmiter RD, Brinster RL, Hammer RE, Trumbauer ME, et al. (1982). Dramatic growth of mice that develop from eggs microinjected with metallothionein-growth hormone fusion genes. Nature 300: 611-616.

Takiguchi M, James C, Josefsson EC, Carmichael CL, et al. (2010). Transgenic, inducible RNAi in megakaryocytes and platelets in mice. J. Thromb. Haemost. 8: 2751-2756.

Wiederschain D, Wee S, Chen L, Loo A, et al. (2009). Single-vector inducible lentiviral RNAi system for oncology target validation. Cell Cycle 8: 498-504. 